\title{
A hole revolution, or are we back where we started?
}

\author{
Oliver Pooley* \\ Oriel College, Oxford, OX1 4 EW, UK \\ 27 September 2005; minor revisions 5 January 2006
}

\begin{abstract}
Doubts are raised concerning Rickles' claim that "an exact analog of the hole argument can be constructed in the loop representation of quantum gravity" (Rickles 2005, 415).

Keywords: Hole argument; Loop quantum gravity; Spin-networks; Substantivalism; Relationalism; General covariance
\end{abstract}

\section{Introduction}

In a recent paper in this journal Rickles investigates the extent to which the hole argument of classical general relativity can be carried over to the formalism of loop quantum gravity. This is a very worthwhile task. Whether or not one is sympathetic to the presuppositions of the original hole argument, and whether or not one is sympathetic to the deflationary way in which many philosophers have sought to respond to it, it is hard to deny that our understanding of the interpretative questions that arise in the context of classical general relativity has greatly benefitted from the hole argument literature. Despite the relative youth and provisional status of the approaches to quantum gravity that are being actively pursued, it is high time that philosophers started asking the same interpretative questions of these new theories. Investigating if and how the hole argument transfers to the new formalisms is one excellent way to do this.

Rickles' conclusion is that there is indeed an "exact analogue" of the hole argument for loop quantum gravity. Moreover, just as the classical hole argument fails to settle the substantivalist-relationalist issue in favour of relationalism, so too the

${ }^{*}$ E-mail address: oliver.pooley@philosophy.ox.ac.uk 
issue is not settled in the context of loop quantum gravity, despite the (nominally) relationalist commitments of the majority of physicists who work on this approach.

I agree with Rickles that the substantivalist-relationalist debate is not so simply resolved in the context of loop quantum gravity as the comments of some physicists might suggest. However, I do not agree that anything like the hole argument can be constructed in the context of its formalism. The key claim in Rickles' argument is that "(the quantum analog of) Einstein's equation cannot determine where spin-networks are in the manifold. Thus, for any specification of initial data ... the Einstein equation will fail to determine the data at $[t>0]$ " (Rickles, 2005, 425). This is seriously misleading in as much as it suggests a comparison with the classical case, where Einstein's equations do not determine 'where' a particular classical metric field satisfying the equations is to be situated on the spacetime manifold. The vital difference is that each such metric field in a given diffeomorphism equivalence class solves the classical equations. In contrast, the spin networks of Rickles' 'quantum gravitational' hole argument do not solve the 'quantum analogues' of Einstein's equations. This, as we will see, completely undermines the claim that a hole argument can be formulated for loop quantum gravity. Before elaborating, I wish to take issue with one or two other claims in Rickles' paper.

\section{The hole argument in classical general relativity}

Before seeking to construct a hole argument for spin-networks, Rickles considers how the hole argument is framed in various formulations of classical general relativity. After discussing the argument in terms of the standard spacetime formulation of the theory, he turns to the constrained Hamiltonian formulation ${ }^{2}$ Ac-

\footnotetext{
${ }^{1}$ In private communication Rickles has pointed out that he views the proper comparison as one between the spin networks of loop quantum gravity and the 3-metric (of a spacelike hypersurface) of the Hamiltonian formulation of classical general relativity. But here too the disanalogies serve to undermine the claim that there can be a hole argument in loop quantum gravity, as I spell out below (p. 9).

${ }^{2}$ I should mention one misleading aspect of Rickles discussion of the hole argument as it applies to the spacetime formulation of general relativity. He writes:

The 'pullback' field $\phi^{*} g$ [of a Lorentzian 4-metric field $g$ ] will generally be different in the sense that, given a global chart on $\mathcal{M}$ with coordinates $\left\{x^{i}\right\}, \phi^{*} g(x) \neq g(x)$. However, it can happen that $\phi^{*} g=g$, i.e. $\phi$ is a spacetime symmetry for $g$, even though $\phi^{*} g(x) \neq g(x)$. When this happens we have the beginnings of a hole argument: there will be many metrics that solve the equations that will give (locally) different results. (Rickles, 2005, 417)
}

This suggests that only some diffeomorphisms $\phi$ are suitable for constructing the hole argument,

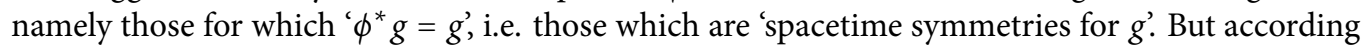
to standard notation and terminology, whenever $\phi$ is a spacetime symmetry, i.e., whenever $\phi^{*} g=g$, it will also be the case that the coordinate components of $g$ and $\phi^{*} g$ are identical relative to every 
cording to Rickles, it is only from the perspective of this formalism that one can properly appreciate the workings of the hole argument, at least qua a problem of determinism (418). This is something of an extraordinary claim given that, as formulated by Earman and Norton (1987), and arguably since its very inception, the hole argument has involved the claim that general relativity, interpreted in certain way, is radically indeterministic. The way in which this indeterminism is manifest in the spacetime formulation (two models of the field equations are identical up to some spacelike surface, but differ to the future of this surface) seems to me to be no less perspicuous than, and, indeed, manifestly equivalent to, the way in which it is manifest in the Hamiltonian formalism (two partially overlapping but divergent curves in the physical phase space both satisfy Hamilton's equations). In fact, one might even argue that the spacetime formalism is more illuminating because it reveals the radical extent of the indeterminism. As Einstein's original 'hole' construction illustrated, and as Earman and Norton stressed, not only does specification of the metric field on the manifold up to some spacelike hypersurface fail to determine the metric properties of any future spacetime points, specification of the field on all of the manifold save some compact region as small as you like fails to determine the metric properties of the points within this region. There is no tidy equivalent of this generalization in the Hamiltonian formalism.

As Rickles nicely sets out, the constrained Hamiltonian formulation of general relativity involves a physical phase space $\mathcal{C}$, the points of which are a 3-dimensional manifold $\Sigma$ equipped with a Riemmanian metric $q_{a b}$ (the configuration variables), and a tensor field $p^{a b}$ (the conjugate momenta). The points of $\mathcal{C}$ represent possible spacelike hypersurfaces of generally relativistic spacetimes, $q_{a p}$ is the 3-metric induced on the hypersurface by the spacetime metric, and $p^{a b}$ is related to the extrinsic curvature of the hypersurface, as it is embedded in spacetime. Not all mathematically possible $\left(q_{a b}, p^{a b}\right)$ pairs are physically possible (in Rickles' notation, the space of all mathematically possible pairs is $\Gamma)$. $\mathcal{C}$ is defined by a set of constraint equations relating $q_{a b}$ and $p^{a b}$; the diffeomorphism constraints $\mathcal{D}_{a}$ (3 equations per space-point) and the Hamiltonian constraint $\mathcal{H}_{\perp}$ (1 equation per space-point).

The constraints are associated with a set of transformations of $\mathcal{C}$ (the gauge transformations), which induce a partition (the gauge orbits) ${ }^{3}$ Rickles claims that

coordinate chart (in Rickles' notation, $\phi^{*} g(x)=g(x)$ ). The truth is that the diffeomorphisms that are spacetime symmetries are precisely the ones that cannot be used to generate two models that present the hole dilemma (i.e., models that agree structurally but that differ in terms of which bit of the structure is exemplified by which part of the manifold). Otherwise the only constraint that a diffeomorphism is required to satisfy if it is to be used in the hole argument is that it reduce to the identity on some suitable region of the manifold (e.g., all of the manifold to the past of some spacelike hypersurface). It is also worth noting that for generic (and realistic) solutions to Einstein's equations, the only $\phi$ such that $\phi^{*} g=g$ is the identity transformation.

${ }^{3}$ Those not already familiar with the relevant mathematics are recommended to consult Belot and Earman 2001, \$\$10.2 and 10.8) before pursuing further references. 
the "relevant constraint for the hole argument is $\mathcal{D}_{a}$ since this generates spatial diffeomorphisms of $\Sigma$. The gauge motions act on all points of $\Gamma$ including those points lying within $\mathcal{C}$, but they have the property of leaving these latter points invariant since they generate gauge transformations on $\mathcal{C}$ " (Rickles, 2005, 419). Later he claims that " $[\mathrm{t}]$ he treatment of the diffeomorphism constraint is the real root of the hole argument for general relativity" (Rickles, 2005, 424). (He qualifies both of these claims in footnotes, in which he admits that the Hamiltonian constraint raises an 'analogous (and deeper) problem' for time and change.)

It seems to me that in some ways this gets things exactly the wrong way round. If the question is simply which transformations within the Hamiltonian formalism correspond to the hole diffeomorphisms of the spacetime formalism, then the answer is, in general, that both those generated by the Hamiltonian and diffeomorphism constraints are needed. ${ }^{4}$ Perhaps a more interesting approach is to pose the analogue of the original hole dilemma within the Hamiltonian framework, without reference to the spacetime picture, and the ontology it suggests.

If we adopt this approach then superficially there is no difference between the transformations generated by the two types of constraint. Both are equally responsible for an apparent breakdown in determinism, because both allow us to transform a curve in $\mathcal{C}$ that solves the Hamiltonian version of Einstein's equations into another curve which partially overlaps the first: an infinity of mathematically distinct solutions pass through a given point in $\mathcal{C}$. It is only when one considers how one should respond to this apparent indeterminism that a difference between the two types of constraint, and the related gauge transformations, emerges. The transformations generated by the diffeomorphism constraint relate distinct points in $\mathcal{C}$, but points that are isometric (they differ solely in terms of which points of $\Sigma$ are assigned which metric properties) . $^{5}$ A very natural interpretative move, therefore,

\footnotetext{
${ }^{4} \mathrm{~A}$ necessary condition for a spacetime diffeomorphism to correspond to a gauge transformation that is generated purely by the diffeomorphism constraint is that it always sends a point of spacetime to a point that is spacelike related to it. This is not a sufficient condition but it at least indicates the rather recherché nature of those hole diffeomorphisms that correspond to a pure diffeomorphism-constraint gauge transformation. Of course, a spacetime diffeomorphism's corresponding to a pure diffeomorphism-constraint gauge transformation is also completely foliation dependent. One and the same diffeomorphism will correspond to a diffeomorphism-constraint gauge transformation with respect to one foliation, but to a transformation involving a Hamiltonianconstraint gauge transformation with respect to another.

${ }^{5}$ As Rickles states, the transformations generated by the diffeomorphism constraints correspond to diffeomorphisms of $\Sigma$ (there is no need for the qualification 'spatial' in this context). But it is not true that these transformations (or, indeed, those generated by the Hamiltonian constraint) leave the points of $\mathcal{C}$ invariant. They do not move one out of a gauge orbit, but within a gauge orbit their action is non trivial. It is only when one passes from $\mathcal{C}$ to the so-called reduced phase space, each point of which corresponds to an entire gauge orbit by construction, that the action of the gauge transformations becomes trivial. Whereas a generally relativistic spacetime corresponds to a curve in $\mathcal{C}$, because every such curve lies entirely within a gauge orbit, a generally relativistic spacetime
} 
is to regard such points as simply mathematically distinct representations of exactly the same physical state, viz. a 3-dimensional space of a particular geometry. One holds that the further distinctions within the formalism, distinctions which mark which point of space has which geometrical property, are artifacts which do not correspond to genuine physical distinctions.

In a sense this exactly parallels a family of responses to the spacetime version of the hole argument, namely those which, one way or another, claim that all diffeomorphic spacetime models represent exactly the same physical state of affairs, a position well-known in the literature as Leibniz Equivalence. There are differences however. One route to Leibniz Equivalence is to question the primitive transmodel identities of the spacetime points that regarding two diffeomorphic models as distinct requires (see Pooley, forthcoming, $\$ \$ 4-5$ ). In the context of the Hamiltonian formalism, our basic points are points of $\Sigma$, a 3-dimensional space. It might seem that taking their repeat appearance in the single manifold that underlies every point of $\mathcal{C}$ also involves taking their trans-temporal identity seriously. For example, consider two initially overlapping curves that solve Hamilton's equations, and are related by a transformation generated the diffeomorphism constraint. A very literalistic interpretation of the formalism might see these curves as representing two possible histories of the very same set of persisting spatial points. These histories involve exactly the same sequence of geometrical relations being instantiated over time. The only way they differ (eventually, when the curves diverge) is in terms of which points instantiate which properties. I hope the absurdity of taking this sort of interpretation seriously is evident. The fact is that physics provides us with no reason to postulate persisting, spatial points, let alone ones that can be re-identified over time entirely independently of the geometrical properties that they instantiate. ${ }^{6}$

The transformations generated by the Hamiltonian constraint on the other hand relate points of $\mathcal{C}$ that correspond to different hypersurfaces of the same generally relativistic spacetime. That is, they correspond to different 3-geometries. If we seek

corresponds simply to a point of the reduced phase space. All curves in $\mathcal{C}$ that lie within the same gauge orbit correspond to the same spacetime. Each particular curve within a gauge orbit corresponds to a particular foliation of the spacetime by hypersurfaces, and particular coordinatizations of each spacelike hypersurface.

${ }^{6}$ It is tempting, therefore, to see the move which identifies points of $\mathcal{C}$ related by the diffeomorphism constraint as a less controversial move than the analogous move in the spacetime context. This would be a mistake. The reason is that someone who does not wish to make the identification in the spacetime context, and who takes the ontology suggested by the spacetime formalism seriously, will obviously not make the analogous identification in the Hamiltonian case either. They will regard the spacetime formalism as fundamental, and will interpret the Hamiltonian formalism in terms of it, i.e., as a way of representing facts about spacetime points rather than persisting spatial points. Hence they will not identify the relevant points of $\mathcal{C}$ even though they regard the identities of the points of $\Sigma$ involved in the mathematical definition of the points of $\mathcal{C}$ as an artifact. 
to evade the apparent indeterminism in this case by regarding the points related by transformations generated by the Hamiltonian constraint as representing the same physical state we are faced with the unpalatable conclusion that the physical state of the world (i.e. 3-space) does not change over time. This is just, as Rickles acknowledges in the footnotes already cited, one version of the well-known problem time in canonical general relativity. The point that I wish to make is that this, rather than anything concerning the transformations generated by the diffeomorphism constraints, is the true heir to the hole argument of the spacetime formalism.

Were it not for the need to construct a quantum theory, I think the solution would be trivial. One simply has to keep in mind the spacetime perspective, and to apply the lessons of the spacetime hole argument, to see that curves in $\mathcal{C}$ related by transformations generated by the Hamiltonian constraint are mathematically distinct representations of the same history, even though the point-by-point pairing of the two curves by the gauge transformation does not relate physically equivalent instantaneous states. The two alternatives, both of which involve taking the 3-dimensional ontology of the Hamiltonian formalism seriously, appear equally unattractive: either the indeterminism is genuine, and pernicious, or there really is no change 7

Before leaving the topic of the classical Hamiltonian framework, I wish to make one final observation about the way in which general relativity differs from some other 'generally covariant', or 'parametrization invariant' Hamiltonian theories. There are two ways in which it might be the case that two curves related by a transformation generated by a Hamiltonian constraint represent the same physical history even though the the point-by-point pairing of the two curves by the gauge transformation does not relate physically equivalent instantaneous states. The first way involves this being the case even though there is some point-by-point correspondence between the two curves that maps physically equivalent states to physically equivalent states. This is the scenario that arises in many of the toy models which are supposed to illustrate the problem of time (for example, Earman, 2002, 8-9). It is true that there is an apparent indeterminism (because the equations of the theory don't determine what the physical state will be at any particular time), but it is equally true that the indeterminism is rather trivial. The sequence of physical states is completely determined; all that is not determined is 'when' each state will occur. The obvious interpretative move to make is to deny the physical meaningfulness of an external time. (The obvious move not to make is to decree that the indeterminism is merely apparent because the transformations generated by the Hamiltonian constraint are genuine gauge transformations, and thus that the states they relate

\footnotetext{
${ }^{7}$ I have previously argued that Barbour's Machian geometrodynamical interpretation of GR (see, e.g., Barbour, 1999. Ch. 11) commits him to the first of these (Pooley, 2002, 130-3; see also Pooley, 2001, \$3.2). The problem does not arise in the conformal generalization of his framework (Anderson et al. 2003).
} 
are really physically equivalent after all. This way lies the problem of time.)

General relativity differs from these simple theories in an important respect. Consider a given foliation of a generally relativistic spacetime, and the corresponding curve in $\mathcal{C}$. Now consider foliating the same spacetime by a different set of spacelike hypersurfaces. This will give us a distinct curve in $\mathcal{C}$ related to the first by a transformation generated by the Hamiltonian constraint. In such a case it is not just the correspondence under the gauge transformation that does not pair off physically equivalent states. No such correspondence exists..$^{8}$ Surely the difficulty of interpreting a quantized version of general relativity, if not the difficulty of constructing such a theory in the first place, is predominantly down to this fact.

\section{A hole argument for quantum general relativity?}

I now turn, finally, to the question of whether anything corresponding to the hole arguments of classical general relativity can be constructed in the context of loop quantum gravity. A very brief recap of the details of the theory is needed.

As Rickles discusses ( $\$ \$_{3}$ and 4 ), loop quantum gravity is a quantization, not of the Hamiltonian version of general relativity that I have so far discussed, but of an alternative formulation involving a different set of fields on $\Sigma$. The old fields ( $q_{a b}$ and $p^{a b}$ ) can be expressed in terms of these new fields. It turns out that the field in terms of which $q_{a b}$ is expressed is actually the momentum variable of the new formalism, and that in terms of which $p^{a b}$ is expressed is the configuration variable. The physical phase space is defined by analogues of the old diffeomorphism and Hamiltonian constraints together with a third family of constraints-the Gauss constraints-that arise because the change of variables involves the introduction of an additional redundancy.

The theory is quantized via Dirac quantization. One starts by considering a space of (suitably behaved) functionals on the space of connections on $\Sigma$. (If one were working with the old variables, one would be considering functionals on the space of 3-metrics on $\Sigma$.) Very roughly, this space of functionals is the kinematical state space, $\mathcal{K}$ (Rovelli, 2004, 226-42). One then promotes the constraint equations to operator equations: the physical state space is the space of those states which are annihilated by (all!) the quantum constraints.

Just as the classical diffeomorphism constraint generated transformations that corresponded to a diffeomorphism of $\Sigma$, so the quantum operator version is the generator of transformations in $\mathcal{K}$ that correspond to diffeomorphisms of $\Sigma$. Thus if a state is annihilated by the diffeomorphism constraint, it is diffeomorphism invariant.

\footnotetext{
${ }^{8}$ For a related discussion, see Wallace (2003).
} 
We are now in a position to evaluate Rickles' quantum gravitational hole argument. He introduces two sets of states: spin-network states $|S\rangle$ and associated sknot states $|s\rangle$. Spin-network states are labelled by certain types of (labelled) graphs (spin networks) embedded in $\Sigma$. Their significance is that they provide a basis for a particular subspace of the kinematical state space $\mathcal{K}$, namely the space of states which satisfies the (quantum) Gauss constraint. They do not, in general, satisfy the diffeomorphism constraint (they do, after all, correspond to embedded graphs). sknot states states, on the other hand, correspond to diffeomorphism equivalence classes of spin networks, and thus are annihilated by the diffeomorphism constraint (as well as by the Gauss constraint).

The key feature of classical general relativity (in both its spacetime and its Hamiltonian guises) that allows for the hole dilemma is that certain transformations are symmetries of the theory (they map solutions of the theory onto solutions), even though they are not (in general) symmetries of the solutions themselves. For example, a generic spacetime model that is a solution of Einstein's field equations will be mapped, by any spacetime diffeomorphism, onto a model that is also a solution. However, this model will (in general) be a mathematically distinct model. Indeed, it must be if the hole dilemma is even to arise, for we need two mathematically distinct models that match in certain respects, and which are both solutions of the theory, for the spectre of indeterminism even to arise ( $c f$. footnote 2 ).

It is exactly this feature that is missing from loop quantum gravity. While diffeomorphisms of $\Sigma$ are symmetries of the theory, they are also symmetries of every (physical) quantum state. That the action of the quantum diffeomorphism constraint on spin-network states is non-trivial is irrelevant. These states are not solutions of theory. Only states that are annihilated by both the Gauss and the diffeomorphism constraints (and, indeed, the quantum Hamiltonian constraint-the equation requiring this is a version of the infamous Wheeler-DeWitt equation) are solutions of the 'quantum analogues of Einstein's equations'. Within the framework of loop quantum gravity, regarding s-knot states, rather than spin-network states, as the genuine physical states is not an optional move that one might be persuaded

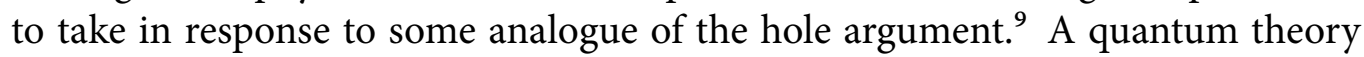
which countenances spin-network states as physical states is simply not a quantum version of general relativity ${ }^{10}$

\footnotetext{
${ }^{9}$ Of course, the real physical states are actually those superpositions of s-knot states that are annihilated by the Hamiltonian constraint.

${ }^{10}$ In fact, the situation I've just described bears a close similarity to that involving the classical and quantum mechanics of identical particles. For the very same reasons, pace Stachel (2002), there is no analogue of the hole argument due to the permutation invariance of non-relativistic quantum mechanics of indistinguishable particles (see Pooley forthcoming). But in general it is not the case that a symmetry of a classical theory becomes a symmetry of the physical states of the corresponding quantum theory (consider the Galilean covariance of non-relativistic quantum mechanics, or the
} 
In the last but one paragraph I considered, for the sake of clarity, the spacetime formulation of the classical theory. As mentioned in footnote 1, Rickles wishes to compare spin-network states to the 3-metrics of the Hamiltonian formalism of classical general relativity (and, presumably, to compare s-knot states to points of superspace-equivalence classes of 3-metrics under 3-diffeomorphisms). I should make it clear why this comparison does not resuscitate Rickles' claim that a hole argument can be constructed in loop quantum gravity.

To recap, the apparent indeterminism in classical Hamiltonian general relativity that is related to the transformations generated by the diffeomorphism constraint arises in the following way. The equations of the theory allow as solutions two curves that up to a certain time pass through the same points in phase space but which then diverge so that one curves passes through one point and the other curve passes through a distinct point but one that nonetheless involve a 3-metric (and extrinsic curvature tensor) related to that of the first point by some 3-diffeomorphism.

What would be a quantum analogue of this situation? Ignore, for the moment, issues connected to the Hamiltonian constraint. An analogue involving spinnetwork states would be a quantum theory that had spin-network states as admissible instantaneous states and which involved evolution equations that, when applied to a given spin-network state as initial data, allowed as possible but exclusive evolutions both of two spin-network states related by a transformation generated by the quantum diffeomorphism constraint.

Taking account of the timeless associated with the Hamiltonian constraint, the analogue becomes a theory that allows as solutions (i.e. as physical states) superpositions of spin-networks that are annihilated by the quantum Hamiltonian constraint but that are not annihilated by the quantum diffeomorphism constraint. It is further required that if a given such state is a solution, then any state obtained from it by transformations generated by the diffeomorphism constraint (with different transformations acting on different spin-network elements of the superposition) should also count as a solution.

It might not be clear whether there could be a quantum theory that picked out just such states as solutions (it would involve an odd kind of indeterministic evolution). It is certainly clear that loop quantum gravity is not such a theory. To the extent that sense can be made of instantaneous states in the theory, they are sknot states, not spin-network states. And to repeat, to be a solution of loop quantum gravity, a state must solve all the constraint equations. Spin-network states do not. But distinct 3-metrics of the classical theory (together with appropriate extrinsic curvature tensors) do solve all the classical constraint equations, and distinct curves passing through such distinct points in phase space can be solutions

Lorentz covariance of relativistic quantum field theory). This particular difference between, e.g., general covariance and Lorentz covariance merits further investigation. 
of all the classical equations. That is why, in the classical case, it must remain an optional interpretative move (albeit an obvious one) to regard equivalence classes of such points as what really correspond to instantaneous states. The corresponding move in loop quantum gravity is already hard-wired into the equations of the theory.

When it comes to loop quantum gravity and the hole argument, then, we are not right back where we started. Indeed, without an understanding why there can be no hole argument in loop quantum gravity, it seems doubtful that progress will be made in providing a coherent interpretation of the theory's ontology ${ }^{11}$

\section{References}

Anderson, E., Barbour, J., Foster, B. and Ó Murchadha, N. (2003), 'Scale-invariant gravity: Geometrodynamics', Classical and Quantum Gravity 20, 1571-604.

Barbour, J. B. (1999), The End of Time: The Next Revolution in Our Understanding of the Universe, Weidenfeld \& Nicholson, London.

Belot, G. and Earman, J. (2001), Pre-Socratic quantum gravity, in C. Callender and N. Huggett, eds, 'Physics Meets Philosophy at the Planck Scale', Cambridge University Press, Cambridge, pp. 213-55.

Earman, J. (2002), 'Thoroughly modern McTaggart', Philosophers' Imprint 2. URL: http://www.philosophersimprint.org/o02003/

Earman, J. and Norton, J. (1987), 'What price spacetime substantivalism? the hole story', British Journal for the Philosophy of Science 38, 515-25.

Pooley, O. (2001), 'Relationalism rehabilitated? II: Relativity', http://philsciarchive.pitt.edu/archive/0000221/

Pooley, O. (2002), The Reality of Spacetime, unpublished D.Phil. thesis.

Pooley, O. (forthcoming), Points, particles and structural realism, in S. French, D. Rickles and J. Saatsi, eds, 'Structural Foundations of Quantum Gravity', Oxford University Press, Oxford.

Rickles, D. P. (2005), 'A new spin on the hole argument', Studies In History and Philosophy of Science Part B: Studies In History and Philosophy of Modern Physics 36, 415-34.

\footnotetext{
${ }^{11}$ I am grateful to Karim Thebault and David Wallace for discussions concerning the interpretation of classical and quantum general relativity.
} 
Rovelli, C. (2004), Quantum Gravity, Cambridge University Press, Cambridge.

Stachel, J. (2002), "the relations between things" versus "the things between relations": The deeper meaning of the hole argument, in D. B. Malament, ed., 'Reading Natural Philosophy. Essays in the History and Philosophy of Science and Mathematics', Open Court, Chicago, pp. 231-66.

Wallace, D. (2003), Time-dependent symmetries: the link between gauge symmetries and indetereminism, in K. Brading and E. Castellani, eds, 'Symmetries in Physics: Philosophical Reflections', Cambridge University Press, Cambridge, pp. 163-73. 